\title{
Boundedness of Fractional Integral Operators Containing Mittag-Leffler Function via Exponentially s-Convex Functions
}

\author{
Gang Hong, ${ }^{1}$ G. Farid, ${ }^{2}$ Waqas Nazeer $\mathbb{D}^{3},{ }^{3}$ S. B. Akbar, ${ }^{4}$ J. Pečarić, ${ }^{5}$ Junzhong Zou $\left(\mathbb{D},{ }^{6}\right.$ \\ and Shengtao Geng $^{7}$ \\ ${ }^{1}$ East University of Heilongjiang, Department of Basic Course, Harbin, Heilongjiang 150066, China \\ ${ }^{2}$ Department of Mathematics, COMSATS University Islamabad, Attock Campus, Islamabad, Pakistan \\ ${ }^{3}$ Department of Mathematics, Government College University, Lahore, Pakistan \\ ${ }^{4}$ Department of Mathematics, COMSATS University Islamabad, Lahore Campus, Islamabad, Pakistan \\ ${ }^{5}$ RUDN University, Moscow, Russia \\ ${ }^{6}$ School of Economics and Management, Harbin Engineering University, Harbin, Heilongjiang 150001, China \\ ${ }^{7}$ College of Electrical and Information Engineering, Zhengzhou University of Light Industry, Zhengzhou 450002, China
}

Correspondence should be addressed to Junzhong Zou; zoujunzhong@163.com

Received 29 January 2020; Accepted 27 March 2020; Published 7 May 2020

Academic Editor: Sei-Ichiro Ueki

Copyright (c) 2020 Gang Hong et al. This is an open access article distributed under the Creative Commons Attribution License, which permits unrestricted use, distribution, and reproduction in any medium, provided the original work is properly cited.

The main objective of this paper is to obtain the fractional integral operator inequalities which provide bounds of the sum of these operators at an arbitrary point. These inequalities are derived for $s$-exponentially convex functions. Furthermore, a Hadamard inequality is obtained for fractional integrals by using exponentially symmetric functions. The results of this paper contain several such consequences for known fractional integrals and functions which are convex, exponentially convex, and $s$-convex.

\section{Introduction}

Convex functions play an important role in many areas of mathematics. They are important especially in the study of optimization problems, theory of inequalities, mathematical analysis, statistical analysis, operation research, and so on. The analytical definition of convex function motivated the authors to define more such functions theoretically; for example, the terms quasi-convex, $m$-convex, $s$-convex, $h$-convex, $(\alpha, m)$-convex, and $(h-m)$-convex functions [1] are defined by extending or generalizing inequality (1). For this paper, we will use exponentially $s$-convex functions which include exponentially convex, $s$-convex, and convex functions.

Definition 1. A function $f: K \subseteq \mathbb{R} \longrightarrow \mathbb{R}$, where $K$ is an interval in $\mathbb{R}$, is said to be convex function if the following inequality holds:

$$
f(t a+(1-t) b) \leq t f(a)+(1-t) f(b),
$$

for all $a, b \in K$ and $t \in[0,1]$.
Definition 2 (see [2]). A function $f: K \subseteq \mathbb{R} \longrightarrow \mathbb{R}$, where $K$ is an interval in $\mathbb{R}$, is said to be exponentially convex function if

$$
f(t a+(1-t) b) \leq t \frac{f(a)}{e^{\alpha a}}+(1-t) \frac{f(b)}{e^{\alpha b}}
$$

holds for all $a, b \in K, t \in[0,1]$ and $\alpha \in \mathbb{R}$. If the inequality in (2) is reversed, then $f$ is called exponentially concave.

A generalization of convex function defined on the right half of the real line is the $s$-convex function defined as follows:

Definition 3 (see [3]). Let $s \in[0,1]$. A function $f:[0, \infty) \longrightarrow \mathbb{R}$ is said to be $s$-convex function in the second sense if

$$
f(t a+(1-t) b) \leq t^{s} f(a)+(1-t)^{s} f(b)
$$

holds for all $a, b \in[0, \infty)$ and $t \in[0,1]$.

It is noted that I-convex function is convex. A further generalization of the above defined functions is given as follows: 
Definition 4 (see [4]). Let $s \in(0,1]$ and $K \subseteq[0, \infty)$ be an interval. A function $f: K \longrightarrow \mathbb{R}$ is said to be exponentially $s$-convex in the second sense if

$$
f(t a+(1-t) b) \leq t^{s} \frac{f(a)}{e^{\alpha a}}+(1-t)^{s} \frac{f(b)}{e^{\alpha b}}
$$

holds for all $a, b \in K, t \in[0,1]$ and $\alpha \in \mathbb{R}$. If the inequality in (4) is reversed, then $f$ is called exponentially $s$-concave.

For utilizations of exponentially convex functions, one can see [2, 4-7]. Our aim in this paper is to utilize exponentially $s$-convex functions for establishing bounds of fractional integral operators with kernel Mittag-Leffler function. The Mittag-Leffler function denoted by $E_{\sigma}(t)$ was introduced by Mittag-Leffler [8] in 1903:

$$
E_{\sigma}(t)=\sum_{n=0}^{\infty} \frac{t^{n}}{\Gamma(\sigma n+1)},
$$

where $\sigma, t \in \mathbb{C}, \Gamma(\cdot)$ is the gamma function, and $\mathfrak{R}(\sigma) \geq 0$.

The Mittag-Leffler function is a direct generalization of the exponential function to which it reduces for $\sigma=1$. In the solution of fractional integral and fractional differential equations, it arises naturally. Due to its importance and utilizations, MittagLeffler function has been generalized by many authors. By direct involvement in the problems of physics, chemistry, biology, engineering, and other applied sciences, Mittag-Leffler function and its generalizations have successful applications. Recently, in [9], Andrić et al. introduced an extended generalized MittagLeffler function which is defined as follows:

Definition 5. Let $\mu, \sigma, l, \gamma, c \in \mathbb{C}, \mathfrak{R}(\mu), \mathfrak{R}(\sigma), \mathfrak{R}(l)>0$, and $\mathfrak{R}(c)>\mathfrak{R}(\gamma)>0$ with $p \geq 0, \delta>0$, and $0<k \leq \delta+\mathfrak{R}(\mu)$. Then, the extended generalized Mittag-Leffler function is defined by

$$
E_{\mu, \sigma, l}^{\gamma, \delta, k, c}(t ; p)=\sum_{n=0}^{\infty} \frac{\beta_{p}(\gamma+n k, c-\gamma)}{\beta(\gamma, c-\gamma)} \frac{(c)_{n k}}{\Gamma(\mu n+\sigma)} \frac{t^{n}}{(l)_{n \delta}},
$$

where $\beta_{p}$ is the generalized beta function defined as follows:

$$
\beta_{p}(x, y)=\int_{0}^{1} t^{x-1}(1-t)^{y-1} e^{-(p / t(1-t))} \mathrm{d} t
$$

and $(c)_{n k}$ is the Pochhammer symbol defined by $(c)_{n k}=(\Gamma(c+n k) / \Gamma(c))$.

From (4), one can obtain the Mittag-Leffler functions defined in [10-14], see Remark 1.3 of [32].

Lemma 1 (see [9]). If $m \in \mathbb{N}$ and $\omega, \mu, \sigma, l, \gamma, c \in \mathbb{C}$ such that $\mathfrak{R}(\mu), \mathfrak{R}(\sigma), \mathfrak{R}(l)>0, \mathfrak{R}(c)>\mathfrak{R}(\gamma)>0$ with $p \geq 0, \delta>0$ and $0<k<\delta+\Re(\mu)$, then

$$
\begin{array}{r}
\left(\frac{\mathrm{d}}{\mathrm{d} t}\right)^{m}\left[t^{\sigma-1} E_{\mu, \sigma, l}^{\gamma, \delta, k, c}\left(\omega t^{\mu} ; p\right)\right]=t^{\sigma-m-1} E_{\mu, \sigma-m, l}^{\gamma, \delta, k, c}\left(\omega t^{\mu} ; p\right), \\
\mathfrak{R}(\sigma)>m .
\end{array}
$$

Fractional integral operators are very useful in mathematical inequalities. \&e authors have established fractional integral inequalities due to different fractional integral operators, see [15-30]. Many authors have used Mittag-Leffler function to define fractional integral operators. The fractional integral operators corresponding to Mittag-Leffler function (4) are defined as follows:

Definition 6 (see [9]). Let $\omega, \mu, \sigma, l, \gamma, c \in \mathbb{C}$, $\mathfrak{R}(\mu), \mathfrak{R}(\sigma), \mathfrak{R}(l)>0$, and $\mathfrak{R}(c)>\mathfrak{R}(\gamma)>0$ with $p \geq 0, \delta>0$, and $0<k \leq \delta+\Re(\mu)$. Let $f \in L_{1}[a, b]$ and $x \in[a, b]$. Then, the generalized fractional integral operators containing MittagLeffler function are defined by

$$
\left(\epsilon_{\mu, \sigma, l, \omega, a^{+}}^{\gamma, \delta, k, c} f\right)(x ; p)=\int_{a}^{x}(x-t)^{\sigma-1} E_{\mu, \sigma, l}^{\gamma, \delta, k, c}\left(\omega(x-t)^{\mu} ; p\right) f(t) \mathrm{d} t,
$$

and

$$
\left(\epsilon_{\mu, \sigma, l, \omega, b^{-}}^{\gamma, \delta, k, c} f\right)(x ; p)=\int_{x}^{b}(t-x)^{\sigma-1} E_{\mu, \sigma, l}^{\gamma, \delta, k, c}\left(\omega(t-x)^{\mu} ; p\right) f(t) \mathrm{d} t,
$$

where $E_{\mu, \sigma, l}^{\gamma, \delta, k, c}(\cdot)$ is the Mittag-Leffler function defined in (6).

From (9) and (10), one can obtain the fractional integral operators defined in [10-14], see Remark 1.4 of [32]. In [20], Farid et al. proved that

$$
\left(\epsilon_{\mu, \sigma, l, \omega, a^{+}}^{\gamma, \delta, k, c} 1\right)(x ; p)=(x-a)^{\sigma} E_{\mu, \sigma+1, l}^{\gamma, \delta, k, c}\left(w(x-a)^{\mu} ; p\right),
$$

and

$$
\left(\epsilon_{\mu, \tau, l, \omega, b^{-}}^{\gamma, \delta, c, c}\right)(x ; p)=(b-x)^{\tau} E_{\mu, \tau+1, l}^{\gamma, \delta, k, c}\left(w(b-x)^{\mu} ; p\right) .
$$

We will follow the upcoming notations in the main results:

$$
\begin{gathered}
D_{\sigma, \omega, a^{+}}(x ; p)=\left(\epsilon_{\mu, \sigma, l, \omega, a}^{\gamma, \delta, k, c} 1\right)(x ; p), \\
D_{\tau, \omega, b^{-}}(x ; p)=\left(\epsilon_{\mu, \tau, l, \omega, b^{-}}^{\gamma, \delta, k, c} 1\right)(x ; p) .
\end{gathered}
$$

The upcoming section contains the main results which address the bounds of fractional integral operators containing Mittag-Leffler functions for exponentially $s$-convex functions. The continuity of such fractional integrals is proved. A Hadamard inequality is established that leads to several Hadamard inequalities for convex, exponentially convex, and $s$-convex functions. Moreover, the results of papers $[31,32]$ can be obtained in particular.

\section{Main Results}

Theorem 1. Let $f:[a, b] \longrightarrow \mathbb{R}$ be a real-valued function. If $f$ is positive and exponentially s-convex, then for $\sigma, \tau \geq 1$, the following upper bound for generalized integral operators holds:

$$
\begin{gathered}
\left(\epsilon_{\mu, \sigma, l, \omega, a^{+}}^{\gamma, \delta, k, c} f\right)(x ; p)+\left(\epsilon_{\mu, \tau, l, \omega, b^{-}}^{\gamma, \delta, k, c} f\right)(x ; p) \\
\leq\left(\frac{f(a)}{e^{\alpha a}}+\frac{f(x)}{e^{\alpha x}}\right) \frac{(x-a) D_{\sigma-1, a^{+}}(x ; p)}{s+1} \\
+\left(\frac{f(b)}{e^{\beta b}}+\frac{f(x)}{e^{\beta x}}\right) \frac{(b-x) D_{\tau-1, b^{-}}(x ; p)}{s+1}, \\
x \in[a, b], \alpha, \beta \in \mathbb{R} .
\end{gathered}
$$


Proof. Let $x \in[a, b]$. Then, for $t \in[a, x)$ and $\sigma \geq 1$, we have the following inequality:

$$
\begin{aligned}
& (x-t)^{\sigma-1} E_{\mu, \sigma, l}^{\gamma, \delta, k, c}\left(\omega(x-t)^{\mu} ; p\right) \\
& \quad \leq(x-a)^{\sigma-1} E_{\mu, \sigma, l}^{\gamma, \delta, k, c}\left(\omega(x-a)^{\mu} ; p\right) .
\end{aligned}
$$

As $f$ is exponentially $s$-convex, therefore, one can obtain

$$
f(t) \leq\left(\frac{x-t}{x-a}\right)^{s} \frac{f(a)}{e^{\alpha a}}+\left(\frac{t-a}{x-a}\right)^{s} \frac{f(x)}{e^{\alpha x}} .
$$

By multiplying (13) and (14) and then integrating over $[a, x]$, we get

$$
\begin{aligned}
\int_{a}^{x}(x-t)^{\sigma-1} E_{\mu, \alpha, l}^{\gamma, \delta, k, c}\left(\omega(x-t)^{\mu} ; p\right) f(t) \mathrm{d} t \\
\leq \frac{(x-a)^{\alpha-1} E_{\mu, \sigma, l}^{\gamma, \delta, c}\left(\omega(x-a)^{\mu} ; p\right)}{(x-a)^{s}} \\
\quad \cdot\left(\frac{f(a)}{e^{\alpha a}} \int_{a}^{x}(x-t)^{s} \mathrm{~d} t+\frac{f(x)}{e^{\alpha x}} \int_{a}^{x}(t-a)^{s} \mathrm{~d} t\right) .
\end{aligned}
$$

The left integral operator follows the upcoming inequality:

$$
\left(\epsilon_{\mu, \sigma, l, \omega, a^{+}}^{\gamma, \delta, k,} f\right)(x ; p) \leq \frac{(x-a) D_{\sigma-1, a^{+}}(x ; p)}{s+1}\left(\frac{f(a)}{e^{\alpha a}}+\frac{f(x)}{e^{\alpha x}}\right) .
$$

Now, on the contrary, for $t \in(x, b]$ and $\tau \geq 1$, we have the following inequality:

$$
\begin{aligned}
& (t-x)^{\tau-1} E_{\mu, \tau, l}^{\gamma, \delta, k, c}\left(\omega(t-x)^{\mu} ; p\right) \\
& \quad \leq(b-x)^{\tau-1} E_{\mu, \tau, l}^{\gamma, \delta, k, c}\left(\omega(b-x)^{\mu} ; p\right) .
\end{aligned}
$$

Again from exponentially $s$-convexity of $f$, we have

$$
f(t) \leq\left(\frac{t-x}{b-x}\right)^{s} \frac{f(b)}{e^{\beta b}}+\left(\frac{b-t}{b-x}\right)^{s} \frac{f(x)}{e^{\beta x}} .
$$

By multiplying (20) and (21) and then integrating over $[x, b]$, we get

$$
\begin{aligned}
\int_{x}^{b}(t-x)^{\tau-1} E_{\mu, \tau, l}^{\gamma, \delta, k, c}\left(\omega(t-x)^{\mu} ; p\right) f(t) \mathrm{d} t \\
\leq \frac{(b-x)^{\tau-1} E_{\mu, \tau, l}^{\gamma, \delta, k, c}\left(\omega(b-x)^{\mu} ; p\right)}{(b-x)^{s}} \\
\quad \cdot\left(\frac{f(b)}{e^{\beta b}} \int_{x}^{b}(t-x)^{s} \mathrm{~d} t+\frac{f(x)}{e^{\beta x}} \int_{x}^{b}(b-t)^{s} \mathrm{~d} t\right) .
\end{aligned}
$$

The right integral operator satisfies the following inequality:

$$
\left(\epsilon_{\mu, \tau, l, \omega, b^{-}}^{\gamma, \delta, c} f\right)(x ; p) \leq \frac{(b-x) D_{\tau-1, b^{-}}(x ; p)}{s+1}\left(\frac{f(b)}{e^{\beta b}}+\frac{f(x)}{e^{\beta x}}\right) .
$$

By adding (19) and (23), required inequality (15) can be obtained.

Corollary 1. If we set $\sigma=\tau$ in (15), then the following inequality is obtained:

$$
\begin{aligned}
& \left(\epsilon_{\mu, \sigma, l, \omega, a^{+}}^{\gamma, \delta, k, c} f\right)(x ; p)+\left(\epsilon_{\mu, \sigma, l, \omega, b^{-}}^{\gamma, \delta, k, c} f\right)(x ; p) \\
& \leq\left(\frac{f(a)}{e^{\alpha a}}+\frac{f(x)}{e^{\alpha x}}\right) \frac{(x-a) D_{\sigma-1, a^{+}}(x ; p)}{s+1} \\
& \quad+\left(\frac{f(b)}{e^{\beta b}}+\frac{f(x)}{e^{\beta x}}\right) \frac{(b-x) D_{\sigma-1, b^{-}}(x ; p)}{s+1}, \quad x \in[a, b] .
\end{aligned}
$$

Corollary 2. Along with assumption of Theorem 1, if $f \in L_{\infty}[a, b]$, then the following inequality is obtained:

$$
\begin{gathered}
\left(\epsilon_{\mu, \sigma, l, \omega, a^{+}}^{\gamma, \delta, k, c} f\right)(x ; p)+\left(\epsilon_{\mu, \tau, l, \omega, b^{-}}^{\gamma, \delta, k, c} f\right)(x ; p) \\
\leq \frac{\|f\|_{\infty}}{s+1}\left[\left(\frac{1}{e^{\alpha a}}+\frac{1}{e^{\alpha x}}\right)(x-a) D_{\sigma-1, a^{+}}(x ; p)\right. \\
\left.+\left(\frac{1}{e^{\beta b}}+\frac{1}{e^{\beta x}}\right)(b-x) D_{\tau-1, b^{-}}(x ; p)\right] .
\end{gathered}
$$

Corollary 3. For $\sigma=\tau$ in (25), we get the following result:

$$
\begin{gathered}
\left(\epsilon_{\mu, \sigma, l, \omega, a^{+}}^{\gamma, \delta, k, c} f\right)(x ; p)+\left(\epsilon_{\mu, \sigma, l, \omega, b^{-}}^{\gamma, \delta, k, c} f\right)(x ; p) \\
\leq \frac{\|f\|_{\infty}}{s+1}\left[\left(\frac{1}{e^{\alpha a}}+\frac{1}{e^{\alpha x}}\right)(x-a) D_{\sigma-1, a^{+}}(x ; p)\right. \\
\left.+\left(\frac{1}{e^{\beta b}}+\frac{1}{e^{\beta x}}\right)(b-x) D_{\sigma-1, b^{-}}(x ; p)\right] .
\end{gathered}
$$

Corollary 4. For $s=1$ in (25), we get the following result for exponentially convex functions:

$$
\begin{gathered}
\left(\epsilon_{\mu, \alpha, l, \omega, a^{+}}^{\gamma, \delta, k, c} f\right)(x ; p)+\left(\epsilon_{\mu, \beta, l, \omega, b^{-}}^{\gamma, \delta, k, c} f\right)(x ; p) \\
\leq \frac{\|f\|_{\infty}}{2}\left[\left(\frac{1}{e^{\alpha a}}+\frac{1}{e^{\alpha x}}\right)(x-a) D_{\alpha-1, a^{+}}(x ; p)\right. \\
\left.+\left(\frac{1}{e^{\beta b}}+\frac{1}{e^{\beta x}}\right)(b-x) D_{\beta-1, b^{-}}(x ; p)\right] .
\end{gathered}
$$

Theorem 2. With the assumptions of Theorem 1, if $f \in L_{\infty}[a, b]$, then operators defined in (9) and (10) are bounded and continuous. 
Proof. If $f \in L_{\infty}[a, b]$, then from (19), we have

$$
\begin{aligned}
& \left|\left(\epsilon_{\mu, \sigma, l, \omega, a^{+}}^{\gamma, \delta, c, c}\right)(x ; p)\right| \\
& \quad \leq \frac{\|f\|_{\infty}(x-a) D_{\sigma-1, a^{+}}(x ; p)}{s+1}\left(\frac{1}{e^{\alpha a}}+\frac{1}{e^{\alpha x}}\right) \\
& \quad \leq \frac{2\|f\|_{\infty}(b-a) D_{\sigma-1, a^{+}}(b ; p)}{(s+1) e^{\alpha a}},
\end{aligned}
$$

that is,

$$
\left|\left(\epsilon_{\mu, \sigma, l, \omega, a^{+}}^{\gamma, \delta, k, c} f\right)(x ; p)\right| \leq M\|f\|_{\infty},
$$

where $M=\left(2(b-a) D_{\sigma-1, a^{+}}(b ; p) /(s+1) e^{\alpha a}\right)$. Therefore, $\left(\epsilon_{\mu, \sigma, l, \omega, a^{+}}^{\gamma, \delta, k, c} f\right)(x ; p)$ is bounded, and also, it is easy to see that it is linear; hence, this is a continuous operator. Also, on the contrary, from (23), one can obtain

$$
\left|\left(\epsilon_{\mu, \tau, l, \omega, b^{-}}^{\gamma, \delta, k, c} f\right)(x ; p)\right| \leq K\|f\|_{\infty},
$$

where $K=\left(2(b-a) D_{\tau-1, b^{-}}(a ; p) /(s+1) e^{\beta a}\right)$. Therefore, $\left(\epsilon_{\mu, \tau, l, c, b^{-}}^{\gamma, \delta, k, c}(x ; p)\right.$ is bounded and also it is linear, hence continuous.

The next result provides boundedness of the left and the right fractional integrals at an arbitrary point for functions whose derivatives in absolute values are exponentially $s$-convex.

Theorem 3. Let $f:[a, b] \longrightarrow \mathbb{R}$ be a real-valued function. If $f$ is differentiable and $\left|f^{\prime}\right|$ is exponentially s-convex, then for $\sigma, \tau \geq 1$, the following fractional integral inequality for generalized integral operators (9) and (10) holds:

$$
\begin{array}{ll}
\mid\left(\epsilon_{\mu, \sigma+1, l, \omega, a^{+}}^{\gamma, \delta, k, c} f\right)(x ; p)+\left(\epsilon_{\mu, \tau+1, l, \omega, b^{-}}^{\gamma, \delta, k, c} f\right)(x ; p) & \\
& -\left(D_{\sigma-1, a^{+}}(x ; p) f(a)+D_{\tau-1, b^{-}}(x ; p) f(b)\right) \mid \\
\leq & \left(\frac{\left|f^{\prime}(a)\right|}{e^{\alpha a}}+\frac{\left|f^{\prime}(x)\right|}{e^{\alpha x}}\right) \frac{(x-a) D_{\sigma-1, a^{+}}(x ; p)}{s+1} \\
& +\left(\frac{\left|f^{\prime}(b)\right|}{e^{\beta b}}+\frac{\left|f^{\prime}(x)\right|}{e^{\beta x}}\right) \frac{(b-x) D_{\tau-1, b^{-}}(x ; p)}{s+1}, \quad x \in[a, b] .
\end{array}
$$

Proof. Let $x \in[a, b]$ and $t \in[a, x]$; by using exponentially $s$-convexity of $\left|f^{\prime}\right|$, we have

$$
\left|f^{\prime}(t)\right| \leq\left(\frac{x-t}{x-a}\right)^{s} \frac{\left|f^{\prime}(a)\right|}{e^{\alpha a}}+\left(\frac{t-a}{x-a}\right)^{s} \frac{\left|f^{\prime}(x)\right|}{e^{\alpha x}} .
$$

From (32), one can have

$$
f^{\prime}(t) \leq\left(\frac{x-t}{x-a}\right)^{s} \frac{\left|f^{\prime}(a)\right|}{e^{\alpha a}}+\left(\frac{t-a}{x-a}\right)^{s} \frac{\left|f^{\prime}(x)\right|}{e^{\alpha x}} .
$$

The product of (16) and (33) gives the following inequality:

$$
\begin{aligned}
& (x-t)^{\sigma-1} E_{\mu, \sigma, l}^{\gamma, \delta, c, c}\left(\omega(x-t)^{\mu} ; p\right) f^{\prime}(t) \mathrm{d} t \\
& \leq(x-a)^{\sigma-1-s} E_{\mu, \sigma, l}^{\gamma, \delta, k, c}\left(\omega(x-a)^{\mu} ; p\right) \\
& \quad \cdot\left(\frac{\left|f^{\prime}(a)\right|}{e^{\alpha a}}(x-t)^{s}+\frac{\left|f^{\prime}(x)\right|}{e^{\alpha x}}(t-a)^{s}\right) .
\end{aligned}
$$

After integrating the above inequality over $[a, x]$, we get

$$
\begin{aligned}
\int_{a}^{x}(x-t)^{\sigma-1} E_{\mu,,, l}^{\gamma, \delta, k, c}\left(\omega(x-t)^{\mu} ; p\right) f^{\prime}(t) \mathrm{d} t \\
\leq(x-a)^{\sigma-1-s} E_{\mu, \sigma, l}^{\gamma, \delta, k, c}\left(\omega(x-a)^{\mu} ; p\right) \\
\quad \cdot\left(\frac{\left|f^{\prime}(a)\right|}{e^{\alpha a}} \int_{a}^{x}(x-t)^{s} \mathrm{~d} t+\frac{\left|f^{\prime}(x)\right|}{e^{\alpha x}} \int_{a}^{x}(t-a)^{s} \mathrm{~d} t\right) \\
=\frac{(x-a)^{\sigma} E_{\mu, \sigma, l}^{\gamma, \delta, k, c}\left(\omega(x-t)^{\mu} ; p\right)}{s+1}\left(\frac{\left|f^{\prime}(a)\right|}{e^{\alpha a}}+\frac{\left|f^{\prime}(x)\right|}{e^{\alpha x}}\right) .
\end{aligned}
$$

The left-hand side of (35) is calculated as follows:

$$
\int_{a}^{x}(x-t)^{\sigma-1} E_{\mu, \sigma, l}^{\gamma, \delta, k, c}\left(\omega(x-t)^{\mu} ; p\right) f^{\prime}(t) \mathrm{d} t .
$$

Putting $x-t=z$, that is, $t=x-z$ and also utilizing (8), we have

$$
\begin{aligned}
& \int_{0}^{x-a} z^{\sigma-1} E_{\mu, \sigma, l}^{\gamma, \delta, k, c}\left(\omega z^{\mu} ; p\right) f^{\prime}(x-z) \mathrm{d} z \\
& =(x-a)^{\sigma-1} E_{\mu, \sigma, l}^{\gamma, \delta, k, c}\left(\omega(x-a)^{\mu} ; p\right) f(a) \\
& \quad-\int_{0}^{x-a} z^{\sigma-2} E_{\mu, \sigma, l}^{\gamma, \delta, c}\left(\omega z^{\mu} ; p\right) f(x-z) \mathrm{d} z .
\end{aligned}
$$

Now, putting $x-z=t$ in the second term of the righthand side of the above equation and then using (9), we get

$$
\begin{aligned}
\int_{0}^{x-a} & z^{\sigma-1} E_{\mu, \sigma, l}^{\gamma, \delta, k, c}\left(\omega z^{\mu} ; p\right) f^{\prime}(x-z) \mathrm{d} z \\
= & (x-a)^{\sigma-1} E_{\mu, \sigma, l}^{\gamma, \delta, k, c}\left(\omega(x-a)^{\mu} ; p\right) f(a) \\
& -\left(\epsilon_{\mu, \sigma+1, l, \omega, a^{+}}^{\gamma, \delta, c} f\right)(x ; p) .
\end{aligned}
$$

Therefore, (35) takes the following form:

$$
\begin{aligned}
& \left(D_{\sigma-1, a^{+}}(x ; p)\right) f(a)-\left(\epsilon_{\mu, \sigma+1, l, \omega, a^{+}}^{\gamma, \delta, c} f\right)(x ; p) \\
& \quad \leq \frac{(x-a) D_{\sigma-1, a^{+}}(x ; p)}{s+1}\left(\frac{\left|f^{\prime}(a)\right|}{e^{\alpha a}}+\frac{\left|f^{\prime}(x)\right|}{e^{\alpha x}}\right) .
\end{aligned}
$$

Also, from (32), one can have

$$
f^{\prime}(t) \geq-\left(\left(\frac{x-t}{x-a}\right)^{s} \frac{\left|f^{\prime}(a)\right|}{e^{\alpha a}}+\left(\frac{t-a}{x-a}\right)^{s} \frac{\left|f^{\prime}(x)\right|}{e^{\alpha x}}\right) .
$$

Following the same procedure as we did for (33), one can obtain 


$$
\begin{aligned}
& \left(\epsilon_{\mu, \sigma+1, l, \omega, a^{+}}^{\gamma, \delta, k, c} f\right)(x ; p)-D_{\sigma-1, a^{+}}(x ; p) f(a) \\
& \quad \leq \frac{(x-a) D_{\sigma-1, a^{+}}(x ; p)}{s+1}\left(\frac{\left|f^{\prime}(a)\right|}{e^{\alpha a}}+\frac{\left|f^{\prime}(x)\right|}{e^{\alpha x}}\right) .
\end{aligned}
$$

From (39) and (41), we get

$$
\begin{aligned}
& \left|\left(\epsilon_{\mu, \sigma+1, l, \omega, a^{+}}^{\gamma, \delta, k, c}\right)(x ; p)-D_{\sigma-1, a^{+}}(x ; p) f(a)\right| \\
& \quad \leq \frac{(x-a) D_{\sigma-1, a^{+}}(x ; p)}{s+1}\left(\frac{\left|f^{\prime}(a)\right|}{e^{\alpha a}}+\frac{\left|f^{\prime}(x)\right|}{e^{\alpha x}}\right) .
\end{aligned}
$$

Now, we let $x \in[a, b]$ and $t \in[x, b]$. Then, by exponentially $s$-convexity of $\left|f^{\prime}\right|$, we have

$$
\left|f^{\prime}(t)\right| \leq\left(\frac{t-x}{b-x}\right)^{s} \frac{\left|f^{\prime}(b)\right|}{e^{\beta b}}+\left(\frac{b-t}{b-x}\right)^{s} \frac{\left|f^{\prime}(x)\right|}{e^{\beta x}} .
$$

On the same lines as we have done for (16), (33), and (40), one can get from (20) and (43) the following inequality:

$$
\begin{aligned}
& \left|\left(\epsilon_{\mu, \tau+1, l, \omega, b^{-}}^{\gamma, \delta, k, c}\right)(x ; p)-D_{\tau-1, b^{-}}(x ; p) f(b)\right| \\
& \quad \leq \frac{(b-x) D_{\tau-1, b^{-}}(x ; p)}{s+1}\left(\frac{\left|f^{\prime}(b)\right|}{e^{\beta b}}+\frac{\left|f^{\prime}(x)\right|}{e^{\beta x}}\right) .
\end{aligned}
$$

From inequalities (42) and (44), via triangular inequality, (28) can be obtained.

The following results hold as special cases.

Corollary 5. If we put $\sigma=\tau$ in (28), then the following inequality is obtained:

$$
\begin{aligned}
& \left(\epsilon_{\mu, \sigma+1, l, \omega, a^{+}}^{\gamma, \delta, k)}\right)(x ; p)+\left(\epsilon_{\mu, \sigma+1, l, \omega, b^{-}}^{\gamma, \delta, k, c} f\right)(x ; p) \\
& -\left(D_{\sigma-1, a^{+}}(x ; p) f(a)+D_{\sigma-1, b^{-}}(x ; p) f(b)\right) \mid \\
& \quad \leq\left(\frac{\left|f^{\prime}(a)\right|}{e^{\alpha a}}+\frac{\left|f^{\prime}(x)\right|}{e^{\alpha x}}\right) \frac{(x-a) D_{\sigma-1, a^{+}}(x ; p)}{s+1} \\
& \quad+\left(\frac{\left|f^{\prime}(b)\right|}{e^{\beta b}}+\frac{\left|f^{\prime}(x)\right|}{e^{\beta x}}\right) \frac{(b-x) D_{\sigma-1, b^{-}}(x ; p)}{s+1}, \quad x \in[a, b] .
\end{aligned}
$$

Definition 7. Let $f:[a, b] \longrightarrow \mathbb{R}$ be a function; we say $f$ is exponentially symmetric about $((a+b) / 2)$ if

$$
\frac{f(x)}{e^{\alpha x}}=\frac{f(a+b-x)}{e^{\alpha(a+b-x)}}, \quad \alpha \in \mathbb{R} .
$$

It is required to give the following lemma which will be helpful to produce Hadamard-type estimations.

Lemma 2. Let $f:[a, b] \longrightarrow \mathbb{R}$ be an exponentially s-convex function. If $f$ is exponentially symmetric, then the following inequality holds:

$$
f\left(\frac{a+b}{2}\right) \leq \frac{f(x)}{2^{s-1} e^{\alpha x}}
$$

Proof. For $[a, b] \subset \mathbb{R}$ be a closed interval, $t \in[0,1]$, and $\alpha \in \mathbb{R}$, we have

$$
f\left(\frac{a+b}{2}\right)=f\left(\frac{a t+(1-t) b}{2}+\frac{a(1-t)+b t}{2}\right) .
$$

Since $f$ is exponentially $s$-convex, so

$$
f\left(\frac{a+b}{2}\right) \leq \frac{f(a t+(1-t) b)}{2^{s} e^{\alpha(a t+(1-t) b)}}+\frac{f(a(1-t)+b t)}{2^{s} e^{\alpha(a(1-t)+b t)}} .
$$

Let $x=a t+(1-t) b$, where $x \in[a, b]$. Then, we have $a+b-x=b t+(1-t) a$, and we get

$$
f\left(\frac{a+b}{2}\right) \leq \frac{f(x)}{2^{s} e^{\alpha(x)}}+\frac{f(a+b-x)}{2^{s} e^{\alpha(a+b-x)}} .
$$

Now, using the fact of exponentially symmetric, we will get (47).

Theorem 4. Let $f:[a, b] \longrightarrow \mathbb{R}, a>b$, be a real-valued function. If $f$ is positive, exponentially s-convex, and symmetric about $((a+b) / 2)$, then for $\sigma, \tau>0$, the following fractional integral inequality for generalized integral operators (9) and (10) holds:

$$
\begin{aligned}
& 2^{s-1} e^{\alpha x} f\left(\frac{a+b}{2}\right)\left[D_{\tau+1, b^{-}}(a ; p)+D_{\sigma+1, a^{+}}(b ; p)\right] \\
& \quad \leq\left(\epsilon_{\mu, \tau+1, l, \omega, b^{-}}^{\gamma, \delta, k, c} f\right)(a ; p)+\left(\epsilon_{\mu, \sigma+1, l, \omega, a^{+}}^{\gamma, \delta, k, c} f\right)(b ; p) \\
& \quad \leq\left[D_{\tau-1, b^{-}}(a ; p)+D_{\sigma-1, a^{+}}(b ; p)\right] \frac{(b-a)^{2}}{s+1}\left(\frac{f(a)}{e^{\alpha a}}+\frac{f(b)}{e^{\beta b}}\right) .
\end{aligned}
$$

Proof. For $x \in[a, b]$, we have

$$
\begin{aligned}
& (x-a)^{\tau} E_{\mu, \tau, l}^{\gamma, \delta, k, c}\left(\omega(x-a)^{\mu} ; p\right) \\
& \quad \leq(b-a)^{\tau} E_{\mu, \tau, l}^{\gamma, \delta, k, c}\left(\omega(b-a)^{\mu} ; p\right), \quad \tau>0 .
\end{aligned}
$$

As $f$ is exponentially s-convex, so for $x \in[a, b]$, we have

$$
f(x) \leq\left(\frac{x-a}{b-a}\right)^{s} \frac{f(b)}{e^{\beta b}}+\left(\frac{b-x}{b-a}\right)^{s} \frac{f(a)}{e^{\alpha a}} .
$$

By multiplying (52) and (53) and then integrating over $[a, b]$, we get

$$
\begin{aligned}
\int_{a}^{b}(x-a)^{\tau} E_{\mu, \tau, l}^{\gamma, \delta, k, c}\left(\omega(x-a)^{\mu} ; p\right) f(x) \mathrm{d} x \\
\leq(b-a)^{\tau-s} E_{\mu, \tau, l}^{\gamma, \delta, k, c}\left(\omega(b-a)^{\mu} ; p\right) \\
\quad \cdot\left(\frac{f(b)}{e^{\beta b}} \int_{a}^{b}(x-a)^{s} \mathrm{~d} x+\frac{f(a)}{e^{\alpha a}} \int_{a}^{b}(b-x)^{s} \mathrm{~d} x\right),
\end{aligned}
$$


from which we have

$$
\begin{aligned}
\left(\epsilon_{\mu, \tau+1, l, \omega, b^{-}}^{\gamma, \delta, k}\right)(a ; p) \leq & \frac{(b-a)^{\tau+1} E_{\mu, \tau, l}^{\gamma, \delta, k, c}\left(\omega(b-a)^{\mu} ; p\right)}{s+1} \\
& \cdot\left(\frac{f(a)}{e^{\alpha a}}+\frac{f(b)}{e^{\beta b}}\right), \\
\left(\epsilon_{\mu, \tau+1, l, \omega, b^{-}}^{\gamma, \delta, k, c} f\right)(a ; p) \leq & \frac{(b-a)^{2}}{s+1} D_{\tau-1, b^{-}}(a ; p)\left(\frac{f(a)}{e^{\alpha a}}+\frac{f(b)}{e^{\beta b}}\right) .
\end{aligned}
$$

Now, on the contrary, for $x \in[a, b]$, we have

$$
\begin{aligned}
& (b-x)^{\sigma} E_{\mu, \sigma, l}^{\gamma, \delta, k, c}\left(\omega(b-x)^{\mu} ; p\right) \\
& \quad \leq(b-a)^{\sigma} E_{\mu, \sigma, l}^{\gamma, \delta, k, c}\left(\omega(b-a)^{\mu} ; p\right), \quad \alpha>0 .
\end{aligned}
$$

By multiplying (53) and (57) and then integrating over $[a, b]$, we get

$$
\begin{aligned}
\int_{a}^{b}(b-x)^{\sigma} E_{\mu, \sigma, l}^{\gamma, \delta, k, c}\left(\omega(b-x)^{\mu} ; p\right) f(x) \mathrm{d} x \\
\leq(b-a)^{\sigma-s} E_{\mu, \sigma, l}^{\gamma, \delta, k, c}\left(\omega(b-a)^{\mu} ; p\right) \\
\quad \cdot\left(\frac{f(b)}{e^{\beta b}} \int_{a}^{b}(x-a)^{s} \mathrm{~d} x+\frac{f(a)}{e^{\alpha a}} \int_{a}^{b}(b-x)^{s} \mathrm{~d} x\right),
\end{aligned}
$$

from which we have

$$
\begin{aligned}
\left(\epsilon_{\mu, \sigma+1, l, \omega, a^{+}}^{\gamma, \delta, k, c} f\right)(b ; p) \leq & \frac{(b-a)^{\sigma+1} E_{\mu, \sigma, l}^{\gamma, \delta, k, c}\left(\omega(b-a)^{\mu} ; p\right)}{s+1} \\
& \cdot\left(\frac{f(a)}{e^{\alpha a}}+\frac{f(b)}{e^{\beta b}}\right),
\end{aligned}
$$

$\left(\epsilon_{\mu, \sigma+1, l, \omega, a^{+}}^{\gamma, \delta, k} f\right)(b ; p) \leq \frac{(b-a)^{2}}{s+1} D_{\sigma-1, a^{+}}(b ; p)\left(\frac{f(a)}{e^{\alpha a}}+\frac{f(b)}{e^{\beta b}}\right)$.

Adding (56) and (60), we get

$$
\begin{aligned}
& \left(\epsilon_{\mu, \tau+1, l, \omega, b^{-}}^{\gamma, \delta, k, c} f\right)(a ; p)+\left(\epsilon_{\mu, \sigma+1, l, \omega, a^{+}}^{\gamma, \delta, k, c} f\right)(b ; p) \\
& \quad \leq\left[D_{\tau-1, b^{-}}(a ; p)+D_{\sigma-1, a^{+}}(b ; p)\right] \frac{(b-a)^{2}}{s+1}\left(\frac{f(a)}{e^{\alpha a}}+\frac{f(b)}{e^{\beta b}}\right) .
\end{aligned}
$$

Multiplying (47) with $(x-a)^{\tau} E_{\mu, \tau, l}^{\gamma, \delta, k, c}\left(\omega(x-a)^{\mu} ; p\right)$ and integrating over $[a, b]$, we get

$$
\begin{aligned}
& f\left(\frac{a+b}{2}\right) \int_{a}^{b}(x-a)^{\tau} E_{\mu, \tau, l}^{\gamma, \delta, k, c}\left(\omega(x-a)^{\mu} ; p\right) \mathrm{d} x \\
& \quad \leq \frac{1}{2^{s-1} e^{\alpha x}} \int_{a}^{b}(x-a)^{\tau} E_{\mu, \tau, l}^{\gamma, \delta, k, c}\left(\omega(x-a)^{\mu} ; p\right) f(x) \mathrm{d} x .
\end{aligned}
$$

By using (10) and (14), we get

$$
f\left(\frac{a+b}{2}\right) D_{\tau+1, b^{-}}(a ; p) \leq \frac{1}{2^{s-1} e^{\alpha x}}\left(\epsilon_{\mu, \tau+1, l, \omega, b^{-}}^{\gamma, \delta, k, c} f\right)(a ; p) .
$$

Multiplying (47) with $(b-x)^{\sigma} E_{\mu, \sigma, l}^{\gamma, \delta, k, c}\left(\omega(b-x)^{\mu} ; p\right)$, integrating over $[a, b]$, and also using $(6)$ and (10), we get

$$
f\left(\frac{a+b}{2}\right) D_{\sigma+1, a^{+}}(b ; p) \leq \frac{1}{2^{s-1} e^{\alpha x}}\left(\epsilon_{\mu, \sigma+1, l, \omega, a^{+}}^{\gamma, \delta, k, c} f\right)(b ; p) .
$$

By adding (63) and (64), we get

$$
\begin{aligned}
& 2^{s-1} e^{\alpha x} f\left(\frac{a+b}{2}\right)\left[D_{\tau+1, b^{-}}(a ; p)+D_{\sigma+1, a^{+}}(b ; p)\right] \\
& \quad \leq\left(\epsilon_{\mu, \tau+1, l, \omega, b^{-}}^{\gamma, \delta, k, c} f\right)(a ; p)+\left(\epsilon_{\mu, \sigma+1, l, \omega, a^{+}}^{\gamma, \delta, k, c} f\right)(b ; p) .
\end{aligned}
$$

By combining (61) and (65), inequality (51) can be obtained.

Corollary 6. If we put $\sigma=\tau$ in (51), then the following inequality is obtained:

$$
\begin{aligned}
& 2^{s-1} e^{\alpha x} f\left(\frac{a+b}{2}\right)\left[D_{\sigma+1, b^{-}}(a ; p)+D_{\sigma+1, a^{+}}(b ; p)\right] \\
& \quad \leq\left(\epsilon_{\mu, \sigma+1, l, \omega, b^{-}}^{\gamma, \delta, k, c} f\right)(a ; p)+\left(\epsilon_{\mu, \sigma+1, l, \omega, a^{+}}^{\gamma, \delta, k, c} f\right)(b ; p) \\
& \quad \leq\left[D_{\sigma-1, b^{-}}(a ; p)+D_{\sigma-1, a^{+}}(b ; p)\right] \frac{(b-a)^{2}}{s+1}\left(\frac{f(a)}{e^{\alpha a}}+\frac{f(b)}{e^{\beta b}}\right) .
\end{aligned}
$$

\section{Concluding Remarks}

We have established the general fractional integral inequalities by using exponentially $s$-convex functions. By selecting particular values at the place of parameters, a variety of results can be obtained. For example, the reader can obtain bounds for fractional integral operators defined by Salim and Faraj in [12] by selecting $p=0$, bounds for fractional integral operators defined by Rahman et al. in [11] by selecting $l=\delta=1$, bounds for fractional integral operators defined by Shukla and Prajapati in [13] by selecting $p=0$ and $l=\delta=1$, bounds for fractional integral operators defined by Prabhakar in [10] by selecting $p=0$ and $l=\delta=k=1$, and bounds for Riemann-Liouville fractional integrals by selecting $p=\omega=0$.

\section{Data Availability}

All the data used to support the findings of this study are included within the article.

\section{Conflicts of Interest}

The authors do not have any conflicts of interest. 


\section{Authors' Contributions}

All authors contributed equally to this paper.

\section{Acknowledgments}

This paper was supported by HEC, Pakistan.

\section{References}

[1] X. Qiang, G. Farid, J. Pecaric, and S. B. Akbar, "Generalized fractional integral inequalities for exponentially $(s, m)$-convex functions," Journal of Inequalities and Applications, vol. 2020, no. 1, pp. 1-13, 2020.

[2] M. U. Awan, M. A. Noor, and K. I. Noor, "Hermite-Hadamard inequalities for exponentially convex functions," Applied Mathematics \& Information Sciences, vol. 12, no. 2, pp. 405-409, 2018.

[3] H. Hudzik and L. Maligranda, "Some remarks on $s$-convex functions," Aequationes Mathematicae, vol. 48, no. 1, pp. 100-111, 1994.

[4] N. Mehreen and M. Anwar, "Hermite-Hadamard type inequalities for exponentially $p$-convex functions and exponentially $s$-convex functions in the second sense with applications," Journal of Inequalities and Applications, vol. 2019, no. 1, p. 92, 2019.

[5] G. Alirezaei and R. Mathar, "On exponentially concave functions and their impact in information theory," in Proceedings of the 2018 Information Theory and Applications Workshop, pp. 11-16, San Diego, CA, USA, February 2018.

[6] S. Pal and T.-K. L. Wong, "Exponentially concave functions and a new information geometry," The Annals of Probability, vol. 46, no. 2, pp. 1070-1113, 2018.

[7] S. Ullah, G. Farid, K. A. Khan, A. Waheed, and S. Mehmood, "Generalized fractional inequalities for quasi-convex functions," Advances in Difference Equations, vol. 2019, p. 15, 2019.

[8] G. M. Mittag-Leffler, "Sur la nouvelle fonction $E_{\alpha}(x)$." Comptes Rendus Hebdomadaires des Séances de l'Académie des Sciences Paris, vol. 137, pp. 554-558, 1903.

[9] M. Andrić, G. Farid, and J. Pečarić, "A further extension of Mittag-Leffler function," Fractional Calculus and Applied Analysis, vol. 21, no. 5, pp. 1377-1395, 2018.

[10] T. R. Prabhakar, "A singular integral equation with a generalized Mittag-Leffler function in the kernel," Yokohama Mathematical Journal, vol. 19, pp. 7-15, 1971.

[11] G. Rahman, D. Baleanu, M. Al Qurashi, S. D. Purohit, S. Mubeen, and M. Arshad, "The extended Mittag-Leffler function via fractional calculus," The Journal of Nonlinear Sciences and Applications, vol. 10, no. 8, pp. 4244-4253, 2017.

[12] T. O. Salim and A. W. Faraj, "A Generalization of MittagLeffler function and integral operator associated with integral calculus," Journal of Fractional Calculus and Applications, vol. 3, no. 5, pp. 1-13, 2012.

[13] A. K. Shukla and J. C. Prajapati, "On a generalization of Mittag-Leffler function and its properties," Journal of Mathematical Analysis and Applications, vol. 336, no. 2, pp. 797-811, 2007.

[14] H. M. Srivastava and Ž. Tomovski, "Fractional calculus with an integral operator containing a generalized Mittag-Leffler function in the kernel," Applied Mathematics and Computation, vol. 211, no. 1, pp. 198-210, 2009.

[15] A. O. Akdemir, A. Ekinci, and E. Set, "Conformable fractional integrals and related new integral inequalities," Journal of Nonlinear and Convex Analysis, vol. 18, no. 4, pp. 661-674, 2017.
[16] P. Citti, M. G. Cowling, and F. Ricci, "Hardy and uncertainty inequalities on stratified Lie groups," Advances in Mathematics, vol. 277, pp. 365-387, 2015.

[17] M. Dokuyucu, D. Baleanu, and E. Çelik, "Analysis of KellerSegel model with Atangana-Baleanu fractional derivative," Filomat, vol. 32, no. 16, pp. 5633-5643, 2018.

[18] M. A. Dokuyucu, E. Celik, H. Bulut, and H. M. Baskonus, "Cancer treatment model with the Caputo-Fabrizio fractional derivative," The European Physical Journal Plus, vol. 133, no. 3, p. 92, 2018.

[19] A. Ekinci and M. E. Ozdemir, "Some new integral inequalities via Riemann Liouville integral operators," Applied and Computational Mathematics, vol. 18, no. 3, pp. 288-295, 2019.

[20] G. Farid, K. A. Khan, N. Latif, A. U. Rehman, and S. Mehmood, "General fractional integral inequalities for convex and $m$-convex functions via an extended generalized Mittag-Leffler function," Journal of Inequalities and Applications, vol. 2018, no. 1, p. 243, 2018.

[21] M. Kunt and I. Iscan, "Fractional hermite hadamard fejer type inequalities for Ga-convex functions," Turkish Journal of Inequalities, vol. 2, no. 1, pp. 1-20, 2018.

[22] A. Kashuri, M. A. Kli, M. Abbas, and H. Budak, "New inequalities for generalized $\mathrm{m}$-convex functions via generalized fractional integral operators and their applications," International Journal of Nonlinear Analysis and Applications, vol. 10, no. 2, pp. 275-299, 2019.

[23] A. Kashuri and R. Liko, "Ostrowski type conformable fractional integrals for generalized $(g, s, m, \varphi)$-preinvex functions," Turkish Journal of Inequalities, vol. 2, no. 2, pp. 54-70, 2018.

[24] D. Nie, S. Rashid, A. O. Akdemir, D. Baleanu, and J.-B. Liu, "On some new weighted inequalities for differentiable exponentially convex and exponentially Quasi-convex functions with applications," Mathematics, vol. 7, no. 8, p. 727, 2019.

[25] M. E. Ozdemir, M. Avci-Ardic, and H. Kavurmaci-Onalan, "Hermite Hadamard type inequalities for $s$-convex and $s$-concave functions via fractional integrals," Turkish Journal of Science, vol. 1, no. 1, pp. 28-40, 2012.

[26] N. Okur and F. B. Yalcin, "Two dimensional operator harmonically convex functions and related generalized inequalities," Turkish Journal of Science, vol. 4, no. 1, pp. 30-38, 2019.

[27] G. Rahman, T. Abdeljawad, F. Jarad, and K. S. Nisar, "Bounds of generalized proportional fractional integrals in general form via convex functions and their applications," Mathematics, vol. 8, no. 1, p. 113, 2020.

[28] S. Rashid, M. A. Noor, and K. I. Noor, "Some new estimates for exponentially $(h, m)$-convex functions via extended generalized fractional integral operators," The Korean Journal of Mathematics, vol. 27, no. 4, pp. 843-860, 2019.

[29] S. Rashid, F. Safdar, A. O. Akdemir, M. A. Noor, and K. I. Noor, "Some new fractional integral inequalities for exponentially m-convex functions via extended generalized Mittag-Leffler function," Journal of Inequalities and Applications, vol. 2019, no. 1, p. 299, 2019.

[30] H. Yaldiz and A. O. Akdemir, "Katugampola fractional integrals within the class of convex functions," Turkish Journal of Science, vol. 3, no. 1, pp. 40-50, 2018.

[31] Y. C. Kwun, G. Farid, W. Nazeer, S. Ullah, and S. M. Kang, "Generalized Riemann-Liouville k -Fractional Integrals Associated With Ostrowski Type Inequalities and Error Bounds of Hadamard Inequalities," IEEE Access, vol. 6, pp. 64946-64953, 2018.

[32] S. M. Kang, G. Farid, W. Nazeer, and M. Usman, "Ostrowski Type Fractional Integral Inequalities for Mappings Whose Derivatives are ( $\alpha, \mathrm{M})$-Convex Via Katugampola Fractional Integrals," Nonlinear Functional Analysis and Applications, vol. 24, pp. 109-126, 2019. 